\title{
Mechanical Insufflator-Exsufflator Maneuver no Collapse, so Says Electrical Impedance Tomography
}

\author{
Milena S Nascimento ${ }^{1}$, Felipe S Rossi ${ }^{1,2}$, Carla F Panonko ${ }^{1}$, Leticia C. Corrêa ${ }^{2}$, Marcelo Amato ${ }^{2,3}$, Celso M Rebello ${ }^{1}$ \\ and Cristiane do Prado ${ }^{1}$
}

${ }^{1}$ Department of Pediatrics, Hospital Israelita Albert Einstein, Brazil

${ }^{2}$ Developer Division, Brazil

${ }^{3}$ Cardiopulmonary Department, Heart Institute (INCOR), Brazil

Submission: April 02, 2020 Published: April 16, 2020

*Corresponding author: Milena S Nascimento, Department of Pediatrics, Hospital Israelita Albert Einstein, São Paulo, Brazil

Abstract

Although mechanical insufflator-exsufflator (MI-E) is widely used among patients with neuromuscular disease, little is known about its effect on alveolar pressure and pulmonary volumes in patients with lung diseases. Currently, outcomes related to MI-E are based mainly on oxygenation, although this parameter is unable to determine secondary collapse or hyper-distension. MI-E application was not yet evaluated by electrical impedance tomography (EIT), a tool that can assess lung aeration during MI-E therapy and enable safety to perform the technique and broaden the application of the procedure for patients with lung disease.

Keywords: Mechanical insufflator-exsufflator; Pediatric; Electrical impedance tomography

Abbreviations: MI-E: Mechanical Insufflator-Exsufflator; EIT: Electrical Impedance Tomography

\section{Introduction}

Mechanical insufflator-exsufflator (MI-E) is a therapy by assisted cough that provide positive and negative pressure to the airways and lead to pulmonary insufflation and exsufflation. This rapid change of pressure produces changes on maximum flow and simulates a natural cough, therefore, moving considerably secretion [1]. Although MI-E is widely used among patients with neuromuscular disease since 1990 [2-4], little is known about this device effect on alveolar pressure, pulmonary volumes and expiratory flow rates in patients with others diseases, and mainly among those with lung diseases [5]. Electrical impedance tomography (EIT) is a non-invasive, portable and easy-to-do procedure on bedside that allows to evaluate regional pulmonary ventilation, cycle-by-cycle, as well as, how to identify changes that occur during real-time ventilation distribution [6]. Currently, outcomes related to non-pharmacological therapy for mucociliary clearance, such as MI-E, or even adjustment of ventilator parameters of invasive mechanical ventilation (IMV) are based on oxygenation, pulmonary complacence and tidal-volume (Vt).
However, these parameters provide a global viewing of lung, and are unable to evaluate regionally if any airways collapsed or suffered a hyper-distension [7]. The evaluation of changes in pulmonary ventilation secondary to MI-E has not yet been evaluated by EIT. Little is known about the alveolar pressures, lung volumes, and expiratory flow rates generated by the MIE in infants and children. The uses of EIT for assessment of response of lung aeration during MI-E therapy enable safety to perform the technique and broaden the application of the procedure for patients with pre-existing lung disease.

\section{Case Report}

Parents provided informed written consent. We report a case of a 16-year-old female patient with Tay-Sachs disease, with advanced encephalopathy, tracheostomized and mechanical ventilation (MV) dependent. Two weeks before hospitalization she deteriorated her ventilatory parameters, with significant reduction of tidal volume (Vt) and turned supplemental oxygen dependent. 
Due to increased tracheal secretion, she was started on antibiotics at home. Two days later, fever and tachycardia developed, and she was hospitalized at the Intensive Care Unit. At admission the patient was hemodynamically stable, the MV parameters were inspiratory pressure of $12 \mathrm{cmH} 20$ over PEEP, PEEP of $6 \mathrm{cmH} 2 \mathrm{O}$, respiratory rate (RR) 20 breaths/min, inspiratory time (Tinsp) 1.0 $\mathrm{sec}$, fraction of inspired oxygen (FiO2) 0.45 , resulting in a Vt of $320 \mathrm{~mL}(5,8 \mathrm{~mL} / \mathrm{kg})$, pressure controlled mode. Initial chest $\mathrm{x}$-ray presented an image suggestive of a lower lobe atelectasis, which was not solved by a bronchoscopy. On the second admission day, the team decided to apply mechanical insufflation-exsufflation (MI-E) therapy to mobilize secretion. As there were some concerns on the effect of negative MI-E pressure in generating pulmonary collapse, electrical impedance tomography (EIT) was used to monitor the procedure (Enlight 1800, Timpel, Sao Paulo, Brazil). EIT is a non-invasive, portable and bedside device that evaluates real-time pulmonary ventilation, identifying changes that occur in ventilation distribution. A pneumotachograph is used in the EIT to match the ventilation data to the image reconstruction, allowing evaluation of functional residual capacity (FRC) and Vt. MI-E therapy used the Cough Assist CA3000 (Philips Respironics, Murrysville, Pennsylvania, United States of America).
The parameters applied during MI-E were inspiratory pressure $+40 \mathrm{cmH} 20$, expiratory pressure $-40 \mathrm{cmH} 20$, inspiratory time 2.0 $\mathrm{sec}$, and expiratory time $3.0 \mathrm{sec}$, without cyclic pause. After the assessment of MI-E by EIT, the patient underwent MI-E during respiratory physiotherapy visits 3 times a day.

\section{Results}

We measured variation in lung ventilation in four regions (upper right, upper left, lower right and lower left). During MI$\mathrm{E}$, a transient loss in FRC was detected in all quadrants but LLQ, where a paradoxical rise was detected (indicating a transient air trapping). The MI-E tidal-volume (Vt) was bigger than $\mathrm{Vt}$ during MV, compatible with the increased pressure gradient set during MI-E (Figure 1). Patent airways were present, even in the collapsed $\mathrm{x}$-ray area, as Vt was detected in the LLQ (Figure 1-panel D). The total lung Vt and FRC are presented in Figure 2, from pre to post MI-E, showing preservation of regional ventilation and endexpiratory lung volume at dependent regions. Thus, contrarily to our expectations, a selective regional trapping of gas may occur during MI-E maneuvers, eventually protecting lung zones at risk. Close monitoring seems essential to confirm that MI-E can be safely applied in unstable patients at risk for lung collapse.
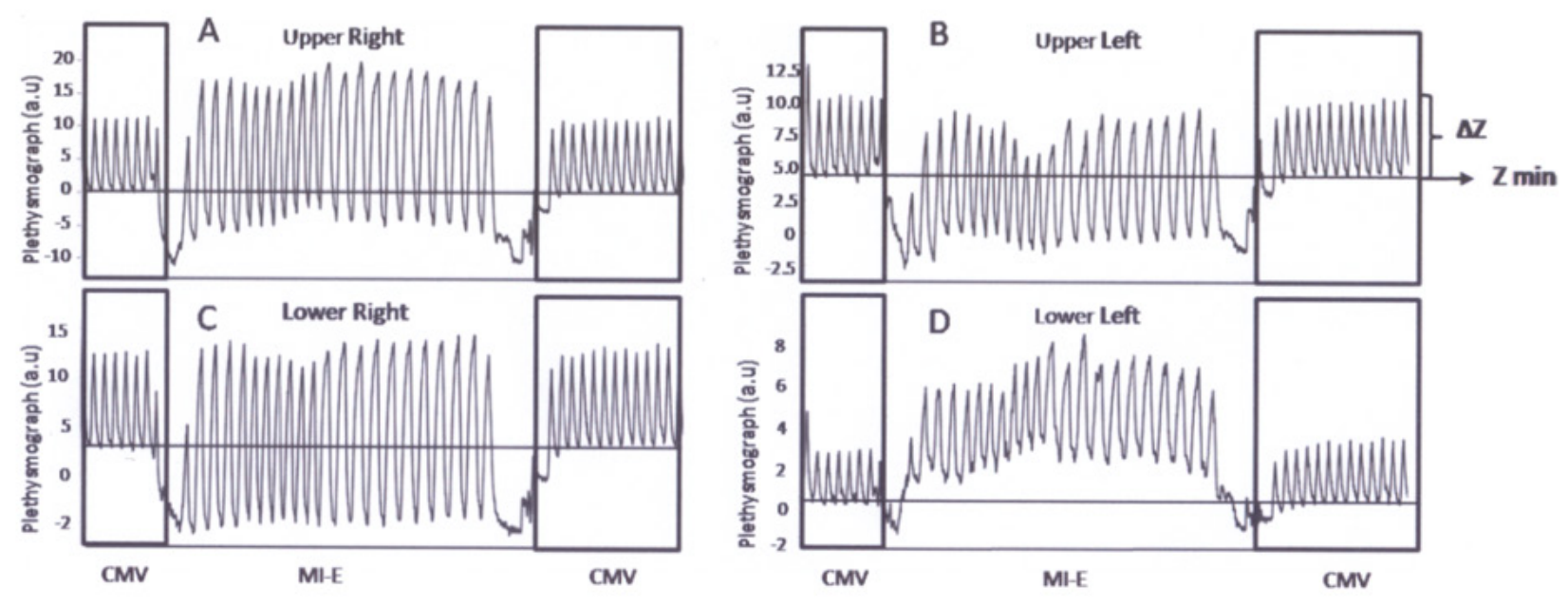

Figure 1: Ventilation variation in four lung quadrants: A (upper right), B (upper left), C (lower right) and D (lower left). The images are divided in three zones. CMV corresponds to the conventional ventilation period, pre and post mechanical insufflation-exsufflation application, which is represented in the middle part (MIE). The $\mathrm{Y}$ axis shows the impedance variation, determined by the EIT plethysmograph, in arbitrary units (a.u), which corresponds to the tidal volume $(\mathrm{Vt})$ variation $(\triangle \mathrm{Z})$. The baseline functional residual capacity (FRC) was underlined in the pre-MIE CMV period, and the line was used to compare FRC (Z min) during the study period. Noticeably, there was a Vt increment during the MIE period, something expected as the pressure gradient during the maneuver is much bigger than during mechanical ventilation. The FRC was transiently diminished during MIE in all quadrants (probably secondary to the negative pressure used), but at the D region, which corresponds to the atelectasis zone. We believe that air could be driven into this area (as Vt change was detected by EIT) but air trapping occurred. A different time constant in this region might relate to this phenomenon.

\section{Discussion}

EIT can evaluate alveolar collapse and FRC changes in critical patients with pulmonary disease [8]. To date, no study has evaluated the effect of a large negative expiratory pressure generated during MI-E using the EIT. Given the lack of in vivo measurements, previous pulmonary model studies suggest that the use of MI-E should be treated with caution, as concerns of possible diseased lung collapse with its use might impact negatively in its application in the clinical practice. The current guidelines indicate 
the use of MI-E in patients who present ineffective cough, with peak flow rates below $270 \mathrm{~L} / \mathrm{min}$, especially in cases of neuromuscular diseases $[9,10]$. Major safety concerns regarding the use of MI-E include risk of barotrauma, collapse of the great airway following application of very negative pressures, and loss of FRC [11]. As shown in the Figure 1, the atelectatic region presented a different FRC response during the MI-E therapy, which we speculate might be related to a different time constant at this region, determining transient air trapping. The remaining regions did present a FRC loss, as a result of the negative pressures applied during MI-E therapy, which was promptly restored after MV resumption. Of note, Vt was detected in all the lung quadrants, which suggests that there were permeable airways even in the area considered collapsed in the chest x-ray. The MI-E higher pressure gradient determined a bigger volume variation in comparison to the MV period, and it was clearly shown in the EIT image (Figure 1). The first MI-E maneuver, the one that was analyzed with EIT, did not impact on a better ventilation profile when comparing dependent and non-dependent zones (Figure 2). In this case report, EIT was capable to show that FRC was only transiently affected by MI-E, but it returned to previous MV situation promptly. This information suggests that the negative pressure of MI-E does not result in sustained alveolar collapse. Unfortunately, only the first MI-E therapy was EIT monitored, but the atelectasis resolution on the next day suggests that no other complication occurred during the following therapies. To our knowledge, only one previous report compared MI-E with conventional physiotherapy in patients with encephalopathy, and the authors found that both techniques rendered efficient results in reverting atelectasis, mobilizing secretion and expanding collapsed areas, but MI-E led to a significantly one day shorter recovery time [12].

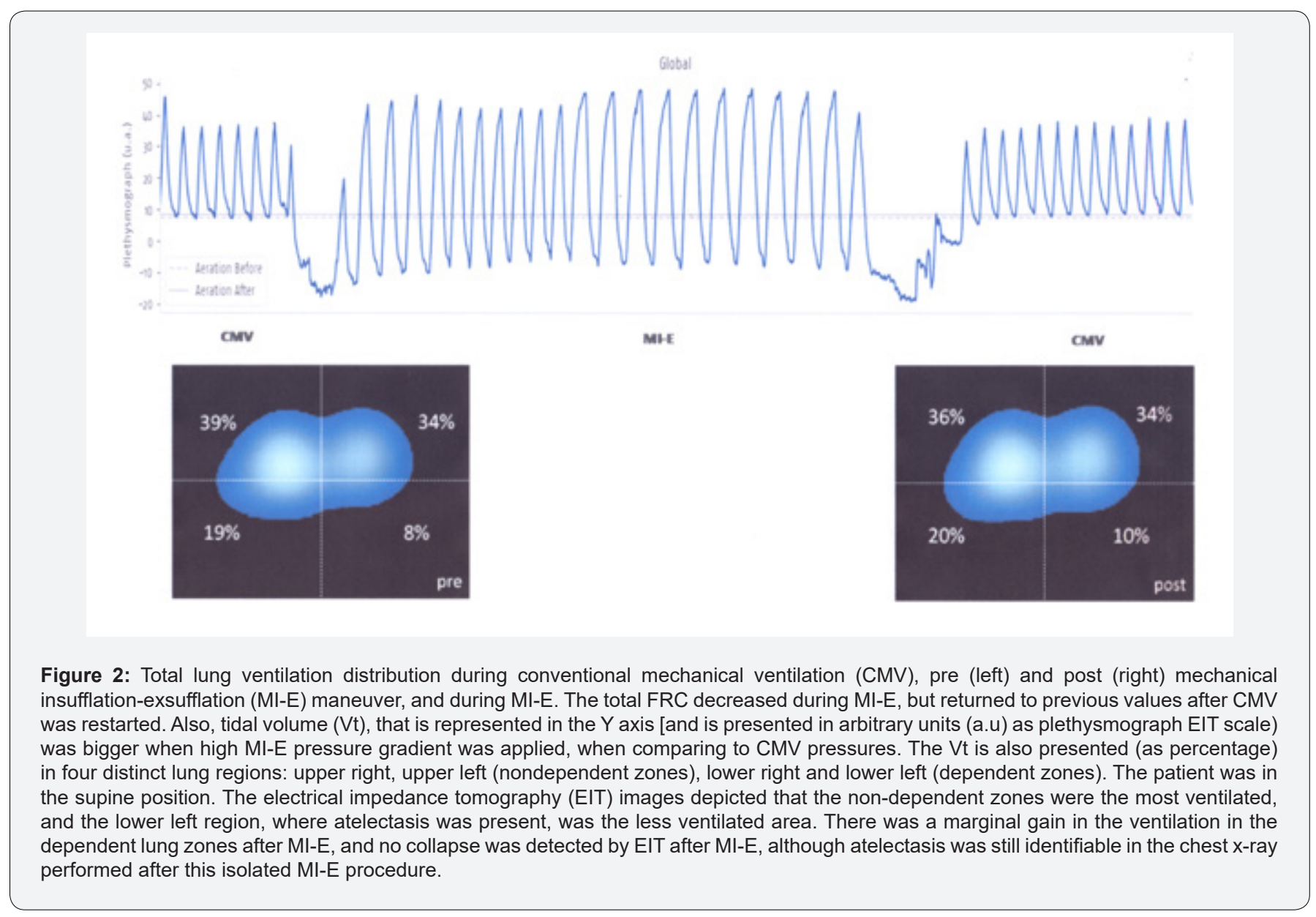

\section{Conclusion}

This report shows that MI-E, when evaluated by EIT, does not promote lung collapse, and can be considered a safe and effective approach to support respiratory physical rehabilitation for bronchial hygiene.

\section{Ethical Approval}

The organization providing ethical approval and ethics protocol reference number where appropriate: 16323519.3.0000.0071 Parents provided informed written consent. 


\section{Conflict of Interest}

Letícia C. Corrêa is employees of Timpel S.A.; Felipe S. Rossi is Timpel S.A. consultant; Marcelo B. P. Amato is Timpel S.A. consultant and minority shareholder. The others authors declare that they have no competing interests.

\section{References}

1. Homnick DN (2007) Mechanical insufflation-exsufflation for airway mucus clearance. Respir Care 52(10): 1296-1305.

2. Bach JR (1993) Mechanical insufflation-exsufflation. Comparison of peak expiratory flows with manually assisted and unassisted coughing techniques. Chest 104(5): 1553-1562.

3. Chatwin M, Ross E, Hart N, Nickol AH, Polkey MI, et al. (2003) Cough augmentation with mechanical insufflation/exsufflation in patients with neuromuscular weakness. Eur Respir J 21(3): 502-508.

4. Homnick DN (2007) Mechanical Insufflation-Exsufflation for Airway Mucus Clearance. Respir Care 52(10): 1296-1305.

5. Winck JC, Goncalves MR, Lourenc,o C, Viana P, Almeida J, et al (2004) Effects of mechanical insufflation-exsufflation on respiratory parameters for patients with chronic airway secretion encumberance. Chest 126(3): 774-780.
6. Barber D, Brown B (1984) Applied potential tomography. J Phys E Sci Instrum 17: 723-733.

7. Luepschen H, Meier T, Grossherr M, Leibecke T, Karsten J, et al. (2007) Protective ventilation using electrical impedance tomography. Physiological Measurement 28(7): 247-260

8. Victorino JA, Borges JB, Okamoto VN, Matos GFJ, Tucci MR, et al. (2004) Imbalances in Regional Lung Ventilation. A Validation Study on Electrical Impedance Tomography. Am J Respir Crit Care Med 169(7): 791-800.

9. Striegl AM, Redding GJ, DiBlasi R, Crotwell D, Salyer J, et al. (2011) Use of a Lung Model to Assess Mechanical In-Exsufflator Therapy in Infants With Tracheostomy. Pediatr Pulmonol 46(3): 211-217.

10. Chatwin M, Ross E, Hart N, Nickol AH, Polkey MI, et al. (2003) Cough augmentation with mechanical insufflation/exsufflation in patients with neuromuscular weakness. Eur Respir J 21(3): 502-508.

11. Fauroux B, Guillemot N, Aubertin G, Nathan N, Labit A, et al. (2008) Physiologic benefits of mechanical insufflation-exsufflation in children with neuromuscular diseases. Chest 133(1):161-168.

12. Siriwat R, Deerojanawong J, Sritippayawan S, Hantragool S, Cheanprapai P (2018) Mechanical Insufflation-Exsufflation Versus Conventional Chest Physiotherapy in Children With Cerebral Palsy. Respir Care 63(2): 187-193.

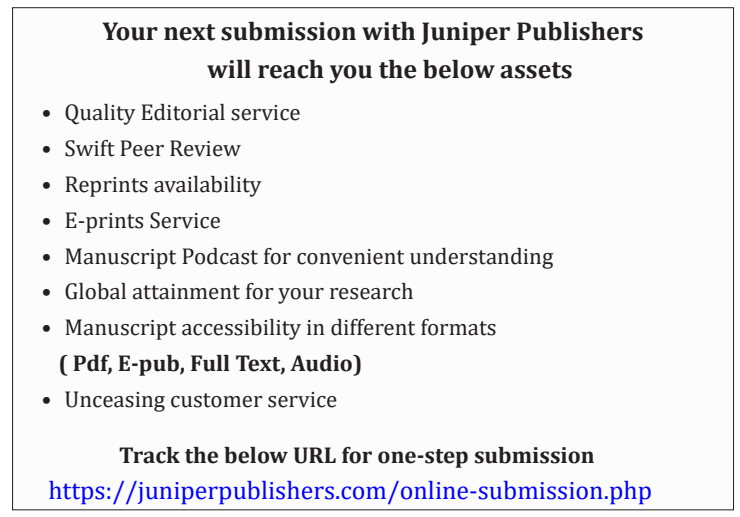

